\title{
SOME PROPERTIES ABOUT COMPLEX DIFFERENCE EQUATIONS OF MALMQUIST TYPE
}

\author{
JIANMING QI
}

Abstract. This article presents versions of the Malmquist type equation. We study the growth of transcendental meromorphic solutions of some complex $(q z+c)$ difference equations and find lower bounds for Nevanlinna lower order for meromorphic solutions of such equations. We also obtain a $(q z+c)$ difference version of Tumura-Clunie theorem, which improves the results of Zheng and Chen[25].

Mathematics subject classification (2010): 30D45, 30D35. equation.

Keywords and phrases: Meromorphic functions, Nevanlinna theory, Malmquist equation, difference

\section{REFERENCES}

[1] M. J. Ablowitz, R. G. Halburd, B. Herbst, On the extension of the Painlevé property to difference equations, Nonlinearly, 13, (2000), 889-905.

[2] D. Barnett, R. G. Halburd, R. J. Korhonen, W. Morgan, Nevanlinna theory for the qdifference equations, Proc. Roy. Soc. Edinburgh Sect. 137, (2007), 457-474.

[3] W. Bergweiler, K. Ishizaki, N. YAnagihara, Meromorphic solutions of some functional equations, Methods Appl. Anal. 5, 3 (1998), 248-259.

[4] W. Bergweiler, J. K. LANGley, Zeros of differences of meromorphic solutions, Math. Proc. Cambridge Philos. Soc. 142, (2007), 133-147.

[5] Y. M. ChIANG, S. J. Feng, On the Nevanlinna characteristic of $f(z+\eta)$ and difference equations in the complex plane, Ramanujan J. 16, (2008), 105-129.

[6] Z. X. CHEN, K. H. SHON, On zeros and fixed points of differences of meromorphic functions, J. Math. Anal. Appl. 344, 1 (2008), 373-383.

[7] J. Clunie, On integral and meromorphic functions, J. London Math. Soc. 37, (1962) 17-27.

[8] V. I. Gromak, I. Laine, S. Shimomura, Painlevé Differential equations in the Complex Plane, Walter de Gruyter, Berlin, 2002.

[9] G. Gudersen, J. Heittokangas, I. Laine, J. Rieppo, D. Yang, Meromorphic solutions of generalized Schröder equation, Aequationes Math. 63, (2002), 110-135.

[10] R. Goldstein, Some results on factorisation of meromorphic functions, J. London Math. Soc. 4, 2 (1971), 357-364.

[11] R. Goldstein, On meromorphic solutions of certain functional equations, Aequationes Math. 18, (1978), 112-157.

[12] R. G. HALBURD, R. J. KORHONEN, Existence of finite-order meromorphic solutions as a detector of integrability in difference equation, Phys. D 218, (2006), 191-203.

[13] R. G. Halburd, R. J. Korhonen, Nevanlinna theory for the difference operator, Ann. Acad. Sci. Fenn. Math. 31, (2006), 463-478.

[14] W. K. Hayman, Meromorphic Functions, Clarendon Press, Oxford, (1964).

[15] W. K. HaYman, On the characteristic of functions meromorphic in the plane and of theire integrals, Proc. London math. Soc. 3, (1963), 93-128.

[16] J. Heittokangas, R. J. Korhonen, I. Laine, J. Rieppo, K. Tohge, Complex difference equations of Malmquist type, Comput. Methods Funct Theory 1, (2001), 27-39. 
[17] K. ISHIZAKI, Hypertranscendency of meromorphic solutions of a linear functional equation, Aequationes Math. 56, (1998), 271-283.

[18] I. Laine, Nevanlinna Theory and Complex Differential Equations, Walter de Gruyter, Berlin, (1993).

[19] I. Laine, J. Rieppo, H. Silvennoinen, Remarks on complex difference equations, Comput. Methods Funct. Theory 5, 1 (2005), 77-88.

[20] R. Nevanlinna, Analytic Function, Springer-Verlag. Berlin/ Heidelberg/ New York, (1970).

[21] A. Ramani, B. Grammaticos, T. Tamizhmani, K. M. Tamizhmani, The road to the discrete analogue of the Painlevé property: Nevanlinna meets singularity confinement, Comput. Math. Appl. 45, (2003), 1001-1012.

[22] Y. Tumura, On the extensions of Borel's theorem and Saxer-Csillag's theorem, Proc. Phys. Math. Soc. Japan 19, (1937), 29-35.

[23] G. Weissenborn, On the theorem of Tumura and Clunie, Bull. London Math. Soc. 18, (1986), 371373.

[24] J. WANG, Growth and poles of meromorphic solutions of some complex difference equations, J. Math. Anal. Appl. 379, (2011), 367-377.

[25] X. M. ZHENG, Z. X. CHEN, Some properties of meromorphic solutions of $q$-difference equations, J. Math. Anal. Appl. 361, (2010), 472-480.

[26] K. LIU, T. B. CAO, Entire solutions of Fermat type q-difference differential equations, Electron. J. Differential Equations 59, (2013), 1-10.

[27] Z. B. Huang, R. R. Zhang, Properties on q-difference Riccati equations, Bull. Korean. Math. Soc. 55, (2018), 1755-1771. 